\title{
Matching random colored points with rectangles
}

\author{
Josué Corujo*1, David Flores-Peñaloza ${ }^{\dagger 2}$, Clemens Huemer ${ }^{\ddagger 3}$, Pablo Pérez-Lantero ${ }^{\S 4}$, and Carlos Seara ${ }^{\llbracket 3}$ \\ ${ }^{1}$ Université Paris-Dauphine, France. Fac. de Matemática y Computación, Universidad de La Habana, Cuba. \\ ${ }^{2}$ Dpto. de Matemáticas, Facultad de Ciencias, Universidad Nacional Autónoma de México, D.F., Mexico. \\ ${ }^{3}$ Dept. de Matemàtiques, Universitat Politècnica de Catalunya, Spain. \\ ${ }^{4}$ Dpto. de Matemática y Ciencia de la Computación, Universidad de Santiago, Santiago, Chile.
}

\begin{abstract}
Let $S \subset[0,1]^{2}$ be a set of $n$ points, randomly and uniformly selected. Let $R \cup B$ be a random partition, or coloring, of $S$ in which each point of $S$ is included in $R$ uniformly at random with probability $1 / 2$. We study the random number $M(n)$ of points of $S$ that are covered by the rectangles of a maximum strong matching of $S$ with axis-aligned rectangles. The matching consists of closed rectangles that cover exactly two points of $S$ of the same color. A matching is strong if all its rectangles are pairwise disjoint. We prove that almost surely $M(n) \geq 0.83 n$ for $n$ large enough. Our approach is based on modeling a deterministic greedy matching algorithm, that runs over the random point set, as a Markov chain.
\end{abstract}

\section{Introduction}

Given a point set $S \subset \mathbb{R}^{2}$ of $n$ points, and a class $\mathcal{C}$ of geometric objects, a geometric matching of $S$ is a set $M \subseteq \mathcal{C}$ such that each element of $M$ contains exactly two points of $S$ and every point of $S$ lies in at most one element of $M$. A geometric matching is strong if the geometric objects are pairwise disjoint, and perfect if every point of $S$ belongs to (or is covered by) some element of $M$. This type of geometric matching problems was considered by Ábrego et al. [1], who studied the existence and properties of matchings for point sets in the plane when $\mathcal{C}$ is the class of axis-aligned squares, or the class of disks.

Let $S=R \cup B \subset \mathbb{R}^{2}$ be a set of $n$ colored points in the plane, each point colored red or blue, where

\footnotetext{
*Email: corujo@ceremade.dauphine.fr.

$\dagger$ Email: dflorespenaloza@gmail.com. Research partially supported by PAPIIT IN117317 (UNAM, Mexico).

‡Email: clemens.huemer@upc.edu. Research supported by projects MTM2015-63791-R (MINECO/FEDER) and Gen. Cat. DGR 2017SGR1336.

§Email: pablo.perez.l@usach.cl. Research supported by project CONICYT FONDECYT/Regular 1160543 (Chile).

đEmail: carlos.seara@upc.edu. Research supported by projects MTM2015-63791-R MINECO/ FEDER and Gen. Cat. DGR 2017SGR1640.
}

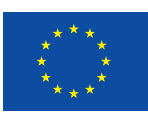

This work has received funding from the European Union's Horizon 2020 research and innovation programme under the Marie SkłodowskaCurie grant agreement No 734922 .
$R$ and $B$ are the sets of red and blue points, respectively. A geometric matching of $S$ is called monochromatic if all matching objects cover points of the same color, and bichromatic if all matching objects cover points of different colors. For example, monochromatic matchings of two-colored point sets in the plane with straight segments have been studied $[4,5]$. In the case of bichromatic matchings with straight segments, a classical result in discrete geometry asserts that for any planar point set $S$ consisting of $n$ red points and $n$ blue points in general position (i.e., no three points of $S$ are collinear) there exists a perfect, strong bichromatic matching of $S$ with straight segments.

In this paper, we consider strong monochromatic matchings with axis-aligned rectangles. Every rectangle will be axis-aligned and a closed set.

Caraballo et al. [2] studied monochromatic strong matchings of $S$ with rectangles from the algorithmic point of view. That is, the problem of finding a monochromatic strong matching of $S$ with the maximum number of rectangles; proving that the problem is NP-hard and giving a polynomial-time 4approximation algorithm. As noted by Caraballo et al., this problem is a special case of the Maximum Independent Set of Rectangles problem (MISR): Given a finite set $\mathcal{R}$ of rectangles in the plane, find a subset $\mathcal{R}^{\prime} \subseteq \mathcal{R}$ of maximum cardinality, denoted $\alpha(\mathcal{R})$, such that every pair of rectangles in $\mathcal{R}^{\prime}$ are disjoint.

Indeed, suppose that we want to find a monochromatic matching of $S$ with the maximum number of rectangles. For every distinct $p, q \in \mathbb{R}^{2}$, let $D(p, q)$ be the minimum axis-aligned rectangle that encloses $p$ and $q$. Let $\mathcal{R}(S)$ be the set of all rectangles $D(p, q)$ such that $p, q \in S, p$ and $q$ have the same color, and $D(p, q)$ contains no points of $S$ different from $p$ and $q$. Finding a monochromatic strong matching of $S$ with the maximum number of rectangles is equivalent to finding in $\mathcal{R}(S)$ a maximum subset of pairwise disjoint rectangles, whose size is $\alpha(\mathcal{R}(S))$. That is, to solving the MISR problem in $\mathcal{R}(S)$.

We study monochromatic strong matchings of $S$ with rectangles from the combinatorial point of view, and from this point forward, every rectangle will cover precisely two points of $S$. Point sets $S=R \cup B$ exist 
in which no matching rectangle is possible (e.g., $S$ is a color-alternating sequence of points on the line $y=x$ ), and point sets in which a perfect strong matching with rectangles exists (e.g., an even number of red points in the negative part of the line $y=x$, and an even number of blue points in the positive part). These two extreme cases show that it is not worth studying the number $\alpha(\mathcal{R}(S))$ for fixed, or given, colored point sets $S$. Instead, we want to study $\alpha(\mathcal{R}(S))$ when $S$ is a random point set in the square $[0,1]^{2}$, in which the positions of the $n$ points of $S$ are random and the color of each point of $S$ is also random. Formally:

Let $n>0$, and let $S \subset[0,1]^{2}$ be a set of $n$ points, randomly and uniformly selected. Let $R \cup B$ be a random partition (i.e., coloring) of $S$ in which each point of $S$ is included in $R$ uniformly at random with probability $1 / 2$. We study the random variable $M(n)=2 \cdot \alpha(\mathcal{R}(S))$ equal to the number of points of $S$ that are covered by the rectangles of a maximum monochromatic strong matching of $S$ with rectangles.

Given a set $S$ of $n$ points, randomly and uniformly selected in the square $[0,1]^{2}$, Chen et al. [3] studied a similar variable: the random variable $\alpha(D(S))$, where $D(S)$ is the random graph with vertex set $S$ and two points $p, q \in S$ define an edge if and only if $D(p, q) \cap S=\{p, q\}$. Here, $\alpha(D(S))$ denotes the size of a maximum independent set of $D(S)$.

One result of Chen et al. [3, Theorem 1] states that if $n$ tends to infinity, then we have $\alpha(D(S))=$ $O\left(n\left(\log ^{2} \log n\right) / \log n\right)$ with probability tending to 1 . This result implies that if $C(n)$ denotes the number of points of $S$ that are covered by a maximum monochromatic matching of $S$ with rectangles, where the rectangles may overlap (i.e., the matching is not necessarily strong), then $C(n)=n-o(n)$ with probability tending to 1 . In fact, let $M^{\prime}$ be a maximum monochromatic matching of $S$ with rectangles, where $M^{\prime}$ is not necessarily strong, and let $S^{\prime} \subset S$ be the points not covered by $M^{\prime}$. Note that at least $\left|S^{\prime}\right| / 2$ points of $S^{\prime}$ have the same color, and they form an independent set in the graph $D(S)$. Then, with probability tending to 1 , we have that $M^{\prime}$ covers at least $n-\left|S^{\prime}\right|=n-O\left(n\left(\log ^{2} \log n\right) / \log n\right)=n-o(n)$ points.

\section{Preliminaries}

Since for matching $S$ with rectangles, only the left-toright and bottom-to-top orders of $S$ are relevant, and since the probability that two points of $S$ are in the same vertical or horizontal line is zero, we consider $S$ equal to the point set $S_{\pi}=\{(i, \pi(i)) \mid i=1,2, \ldots, n\}$, where $\pi:\{1,2, \ldots, n\} \rightarrow\{1,2, \ldots, n\}$ is a randomly and uniformly selected permutation. This assumption was also done by Chen et al. [3].

We have implemented a program that, given $n$, generates a uniform random permutation $\pi$, and selects the color of each $p \in S_{\pi}$ (red or blue) randomly and

\begin{tabular}{|c|c|c|c|c|}
\cline { 2 - 5 } \multicolumn{1}{c|}{} & \multicolumn{2}{c|}{$n=1000$} & \multicolumn{2}{c|}{$n=10000$} \\
\hline$k$ & mean & sdev & mean & sdev \\
\hline 1 & 0.6653 & 0.0175 & 0.6673 & 0.0052 \\
\hline 2 & 0.7948 & 0.0104 & 0.7934 & 0.0036 \\
\hline 3 & 0.8301 & 0.0097 & 0.8304 & 0.0034 \\
\hline 4 & 0.8555 & 0.0094 & 0.8562 & 0.0028 \\
\hline 5 & 0.8727 & 0.0090 & 0.8736 & 0.0026 \\
\hline 6 & 0.8860 & 0.0087 & 0.8864 & 0.0026 \\
\hline$\infty$ & 0.9724 & 0.0062 & 0.9780 & 0.0022 \\
\hline
\end{tabular}

Table 1: The experimental results obtained when running the greedy matching algorithm for $n \in\{1000,10000\}$, parameterized with $k \in[1 . .6]$, or not parameterized $(k=\infty)$. For each combination $n, k$, we run the algorithm 100 times, and measured the mean and standard deviation of the ratio between the total number of matched points and $n$.

uniformly. The program then runs a deterministic algorithm on $S_{\pi}=R \cup B$ that greedily finds a maximum independent subset of rectangles in $\mathcal{R}\left(S_{\pi}\right)$. The algorithm iterates the points of $S_{\pi}$ from left to right, and for each point $p$ in the iteration, it performs the following action: If $p$ is not matched with any point prior to $p$ in the iteration, it finds the first point $q$ to the right of $p$ such that $D(p, q) \in \mathcal{R}\left(S_{\pi}\right)$ and $D(p, q)$ has empty intersection with all matching rectangles already reported. If $q$ exists, the algorithm reports $D(p, q)$ as a matching rectangle. In any case, regardless of whether $q$ exists, the algorithm continues the iteration to the next unmatched point $p$.

For large $n$, say $n=10000$, the implemented algorithm reports a matching covering approximately $\frac{97}{100} n$ of the points. Then, it seems that $M(n) \geq \frac{97}{100} n$ for $n$ large enough and probability close to 1 . Analyzing the algorithm, when run over the random $S_{\pi}$, seems to be a good approach for obtaining a high lower bound for $M(n)$. One way to analyze the algorithm is to consider a parameterized version of it, with a parameter $k$, such that each unmatched point $p$ finds its match point $q$ among only the next $k$ points of $S_{\pi}$ to the right of $p$. Let $\mathcal{A}_{k}$ denote this parameterized algorithm. For experimental results, see Table 1.

We show how to model (an adaptation of) $\mathcal{A}_{k}$ as a Markov chain, for any $k \in\{1,2, \ldots\}$. Then, we show that $\mathcal{A}_{3}$ almost surely guarantees $M(n) \geq \frac{83}{100} n$, for $n$ large enough, by computing the stationary distribution of the Markov chain and applying the Ergodic theorem. See [6] for the theory on Markov chains.

\section{The Markov chains}

We consider $S=S_{\pi}$, and whenever we say point $i$, for $i \in\{1,2, \ldots, n\}$, or just $i$ when it is clear from the context, we are referring to the point $p_{i}:=(i, \pi(i)) \in$ $S$. Let color $(i) \in\{R, B\}$ denote the color of point $i$.

Let $k \in\{1,2,3, \ldots\}$ be a constant, and let $\tilde{\mathcal{A}}_{k}$ be the following adaptation of algorithm $\mathcal{A}_{k}$, consisting 
in the next idea: Suppose that $\mathcal{A}_{k}$ matches points $i$ and $j$, with $i<j \leq i+k$, when the iteration of $S_{\pi}$ is on point $i$. $\tilde{\mathcal{A}}_{k}$ iterates $S_{\pi}$ from left to right, and will also match $i$ and $j$ but, in contrast with $\mathcal{A}_{k}$, when the iteration is on $j$, or on a point to the right of $j$. Using $\tilde{\mathcal{A}}_{k}$ instead of $\mathcal{A}_{k}$, allows us to describe in a more compact way the states of the memory of the algorithm during the iteration of the elements of $S_{\pi}$.

Let $E(j)$ be the data structure associated with point $j \in\{1,2, \ldots, n\}$, that is maintained by $\tilde{\mathcal{A}}_{k}$ during the iteration of $S_{\pi}$. For any $j$, let $i=i(j)$ be the smallest element in the set $\{\max (1, j-(k-1)), \ldots, j\}$ such that the point $i$ is not matched, and each point in $\{i+1, \ldots, j\}$ is matched with a point to the left of $i$ or is not yet matched. If $i$ exists, then $E(j)$ consists of the following elements:

- The set $U(j) \subseteq\{i, i+1, \ldots, j\}$ of the points that are not matched, with $i \in U(j)$.

- The set $\operatorname{Rect}(j)$ of the (pairwise disjoint) rectangles that match the points in $\{i+1, \ldots, j\} \backslash U(j)$ with points to the left of $i$.

- The number $f(j)$ of points of $S_{\pi}$ that are matched while the iteration is at point $j$.

If $i$ does not exist, then $E(j)$ consists of the same three above elements with $U(j)=\emptyset$ and $\operatorname{Rect}(j)=\emptyset$.

For $j=1$, we have $U(1)=\{1\}$, $\operatorname{Rect}(1)=\emptyset$, and $f(1)=0$. We show now how to obtain $E(j+1)$ from $E(j)$, for any $j \in\{1, \ldots, n-1\}$. First, we match points $i$ and $j+1$ if and only if $j+1 \leq i+k$, $\operatorname{color}(i)=\operatorname{color}(j+1)$, and the rectangle $D\left(p_{i}, p_{j+1}\right)$ does not overlap any rectangle in $\operatorname{Rect}(j)$. After that, we match other points in $(U(j) \backslash\{i\}) \cup\{j+1\}$ if and only if $i$ was matched in the previuos step, or we have finished with point $i$. We say that we have finished with point $i$ if there do not exist more chances for point $i$ to be matched, which is equivalent to $i+k \leq j+1$. This final matching procedure consists in running the original algorithm $\mathcal{A}_{k}$ with input the points $\{i+1, \ldots, j, j+1\}$, but with the extra condition that the algorithm terminates if the current point $t$ on the iteration of $\{i+1, \ldots, j, j+1\}$ from left to right, cannot be matched with any other one to its right. This is because $t$ must find its match among the points in $\{j+2, \ldots, t+k\}$, before any matching between points in $\{t+1, \ldots, j+1\}$ occurs. We set $f(j+1)$ equal to the total number of points matched in the above steps. Obtaining $U(j+1)$ and $\operatorname{Rect}(j+1)$ is straightforward.

Let $j \in\{1,2, \ldots, n\}$. The state of $E(j)$ is the 2tuple formed by: As first component, (a certificate of) the relative positions between the points of $U(j)$ and the rectangles of $\operatorname{Rect}(j)$, together with the color of each point of $U(j)$. If the leftmost point is blue, then we switch the color of every point such that the leftmost one is always red. As second component, $f(j)$. We say that two states $e$ and $e^{\prime}$ are equal (i.e., $e=e^{\prime}$ )

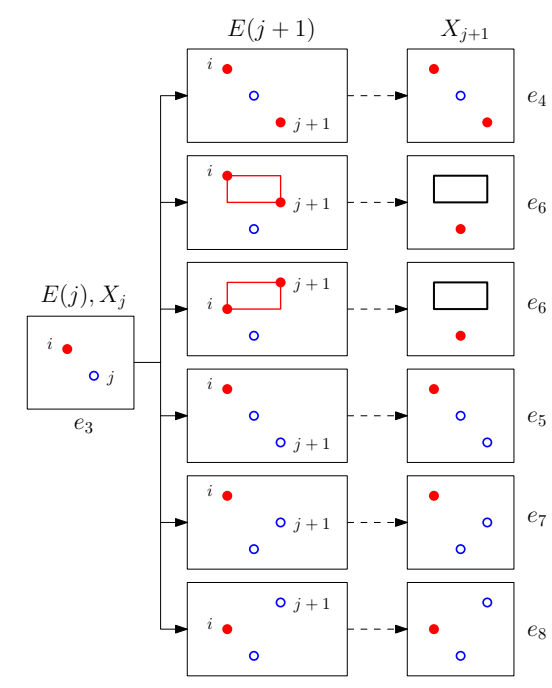

Figure 1: Example of the data structure $E(j)$, its state $X_{j}=e_{3}$, and the states in the neighborhood $N\left(e_{3}\right)=$ $\left\{e_{4}, e_{5}, e_{6}, e_{6}, e_{7}, e_{8}\right\}$ corresponding to $E(j+1)$, for each position and color of point $j+1$. Note that $f\left(e_{6}\right)=2$, and $f(e)=0$ for all $e \in\left\{e_{4}, e_{5}, e_{7}, e_{8}\right\}$.

if: (i) the first components are equal, or one first component is symmetric to the other in the vertical direction, and $(i i)$ the second components are equal.

Let $\mathcal{E}=\left\{e_{1}, e_{2}, \ldots, e_{N}\right\}$ be the set of all possible states of $E(j)$, which is a finite set, and let $X_{j} \in \mathcal{E}$ be the random variable equal to the state of $E(j)$. Let $e \in \mathcal{E}$ be a state, and assume that $e$ is the state of $E(j)$ for some $j$. Let $f(e)=f(j)$ (with abuse of notation), and let $N(e)$ be the neighborhood of $e$, which is the multiset consisting of the state of $E(j+1)$ for every color and every different relative position, with respect to the elements of both $U(j)$ and $\operatorname{Rect}(j)$, of point $j+1$. See for example Figure 1 .

Lemma 1 Let $e, e^{\prime} \in \mathcal{E}$ be two states. For every $j \geq 2$, we have:

$$
\operatorname{Prob}\left(X_{j+1}=e^{\prime} \mid X_{j}=e\right)=\frac{m}{2(|U(j)|+2|\operatorname{Rect}(j)|+1)},
$$

where $m$ is the multiplicity of $e^{\prime}$ in $N(e)$.

Proof. Through each point of $U(j)$ draw a horizontal line, and for each rectangle of $\operatorname{Rect}(j)$ draw a horizontal line through the top side and a horizontal line through the bottom side. Each of these $K=|U(j)|+2|\operatorname{Rect}(j)|$ lines goes through a different element of $S_{\pi}$, subdividing the plane into $K+1$ strips. Since the point $j+1$ is to the right of every point of $U(j)$ and every rectangle of $\operatorname{Rect}(j)$, its relative position w.r.t. the elements of $U(j)$ and $\operatorname{Rect}(j)$ is to be in one of these strips, and this happens with probability $1 /(K+1)$. Furthermore, the color of point $j+1$ is given with probability $1 / 2$. The lemma follows.

Note that $\operatorname{Prob}\left(X_{j+1}=x_{j+1} \mid X_{j}=x_{j}, \ldots, X_{1}=\right.$ $\left.x_{1}\right)=\operatorname{Prob}\left(X_{j+1}=x_{j+1} \mid X_{j}=x_{j}\right)$ for all 
$x_{1}, \ldots, x_{j+1} \in \mathcal{E}$ such that $\operatorname{Prob}\left(X_{j}=x_{j}, \ldots, X_{1}=\right.$ $\left.x_{1}\right)>0$. Thus, $\left(X_{n}\right)_{n \geq 1}$ is a Markov chain, denoted $\mathcal{C}_{k}$, over the set $\mathcal{E}=\left\{e_{1}, e_{2}, \ldots, e_{N}\right\}$ of states. Let $P$ be the transition matrix, of dimensions $N \times N$, such that $P_{i, j}=\operatorname{Prob}\left(X_{\ell+1}=e_{j} \mid X_{\ell}=e_{i}\right)$. The key observation is that the total number of points matched by $\tilde{\mathcal{A}}_{k}$ is precisely $M_{k}(n):=\sum_{j=1}^{n} f\left(X_{j}\right)$.

A Markov chain is irreducible if with positive probability any state can be reached from any other state [6]. It can be proved that $\mathcal{C}_{k}$ is irreducible. Since $\mathcal{C}_{k}$ has a finite state set, it has a unique stationary distribution $s=\left(s_{1}, s_{2}, \ldots, s_{N}\right)$, which is the solution of the system $s=s \cdot P, \quad s_{1}+s_{2}+\cdots+s_{N}=1$ of linear equations [6]. Furthermore, since $f(e) \in$ $\left\{0,2,4, \ldots, 2\left\lceil\frac{k+1}{2}\right\rceil\right\}$ for all $e \in \mathcal{E}$, the function $f$ is bounded and then the Ergodic theorem ensures

$$
\lim _{n \rightarrow \infty} \frac{M_{k}(n)}{n}=\lim _{n \rightarrow \infty} \frac{1}{n} \sum_{j=1}^{n} f\left(X_{j}\right)=\sum_{i=1}^{N} s_{i} f\left(e_{i}\right),
$$

almost surely [6]. Let $\alpha_{k}=\sum_{i=1}^{N} s_{i} f\left(e_{i}\right)$. We then arrive to the main result of this paper:

Theorem 2 Let $\pi:\{1,2, \ldots, n\} \rightarrow\{1,2, \ldots, n\}$ be a uniform random permutation. Let $S_{\pi}=\{(i, \pi(i)) \mid$ $i=1,2, \ldots, n\}$ be a random point set, where the color (red or blue) of each point of $S_{\pi}$ is selected randomly and uniformly with probability $1 / 2$. Let $k \in\{1,2,3, \ldots\}$ be a constant. For all constant $\varepsilon>0$ and $n$ large enough, almost surely the number $M_{k}(n)$ of points of $S_{\pi}$ that are matched by the algorithm $\tilde{\mathcal{A}}_{k}$ satisfies $M_{k}(n) \geq\left(\alpha_{k}-\varepsilon\right) n$.

\section{The Markov chain for $k=3$}

Using algorithm $\tilde{\mathcal{A}}_{3}$, we give a precise value for $\alpha_{3}$. In Table 2, we describe the states, and the transitions between the states, of the Markov chain $\mathcal{C}_{3}$. Since $f(e)=2$ for all $e \in\left\{e_{2}, e_{6}, e_{9}, e_{10}, e_{16}, e_{17}, e_{18}\right\}$, $f\left(e_{11}\right)=4, f(e)=0$ for all other state $e$, and the stationary distribution $s=\left(s_{1}, \ldots, s_{18}\right)$ satisfies

$$
\begin{gathered}
s_{2}=\frac{167959}{816233}, s_{6}=\frac{69640}{816233}, s_{9}=\frac{6800}{816233}, s_{10}=\frac{58650}{816233}, \\
s_{11}=\frac{13600}{816233}, s_{16}=\frac{5950}{816233}, s_{17}=\frac{1360}{816233}, s_{18}=\frac{1190}{816233},
\end{gathered}
$$

we obtain

$$
\begin{aligned}
\alpha_{3} & =2\left(s_{2}+s_{6}+s_{9}+s_{10}+s_{16}+s_{17}+s_{18}\right)+4 s_{11} \\
& =\frac{677498}{816233} \approx 0.830030151 .
\end{aligned}
$$

By Theorem 2, taking $\varepsilon=\alpha_{3}-0.83>0$, for $n$ large enough we have almost surely that $M(n) \geq M_{3}(n) \geq$ $0.83 n$. It can be noted in Table 1 that in practice this lower bound is satisfied.

\section{References}

[1] B. M. Ábrego, E. M. Arkin, S. Fernández-Merchant, F. Hurtado, M. Kano, J. S. Mitchell, and J. Urrutia. Matching points with squares. Discrete \& Computational Geometry, 41(1):77-95, 2009.

\begin{tabular}{|c|c|c|c|}
\hline$e_{i}$ & elem. of $e_{i}$ & $f\left(e_{i}\right)$ & neighbors of $e_{i}$ \\
\hline$e_{1}$ & $\bullet$ & 0 & $\left(e_{2}, 1 / 2\right),\left(e_{3}, 1 / 2\right)$ \\
\hline$e_{2}$ & $\emptyset$ & 2 & $\left(e_{1}, 1\right)$ \\
\hline$e_{3}$ & $\bullet$ & 0 & $\left(e_{4}, 1 / 6\right),\left(e_{5}, 1 / 6\right),\left(e_{6}, 1 / 3\right)$, \\
\hline$e_{4}$ & $\bullet$ & 0 & $\left(e_{4}, 1 / 6\right),\left(e_{8}, 1 / 6\right),\left(e_{5}, 1 / 8\right),\left(e_{6}, 3 / 8\right)$, \\
\hline$e_{5}$ & $\bullet$ & 0 & $\left(e_{7}, 1 / 8\right),\left(e_{9}, 1 / 4\right)$ \\
\hline$\left.e_{6}, 3 / 4\right),\left(e_{11}, 1 / 4\right)$
\end{tabular}

Table 2: The 18 states of the Markov chain for $k=3$. In the 2nd column we show the first component of $e_{i}$, and in the 3 rd column the second component $f\left(e_{i}\right)$. In the last column we show the neighbor states of $e_{i}$ as a list of tuples of the form $\left(e_{j}, P_{i, j}\right)$, where $P_{i, j}=\operatorname{Prob}\left(X_{\ell+1}=e_{j} \mid X_{\ell}=\right.$ $\left.e_{i}\right)>0$ is the transition probability from $e_{i}$ to $e_{j}$.

[2] L. E. Caraballo, C. Ochoa, P. Pérez-Lantero, and J. Rojas-Ledesma. Matching colored points with rectangles. Journal of Combinatorial Optimization, 33(2):403-421, 2017.

[3] X. Chen, J. Pach, M. Szegedy, and G. Tardos. Delaunay graphs of point sets in the plane with respect to axis-parallel rectangles. Random Structures and Algorithms, 34(1):11-23, 2009.

[4] A. Dumitrescu and R. Kaye. Matching colored points in the plane: Some new results. Computational Geometry, 19(1):69-85, 2001.

[5] A. Dumitrescu and W. L. Steiger. On a matching problem in the plane. Discrete Mathematics, 211:183195,2000

[6] J. R. Norris. Markov chains. Number 2. Cambridge University Press, 1998. 